\title{
Near-infrared jet emission in the microquasar XTE J1550-564^
}

\author{
S. Chaty ${ }^{1}$, G. Dubus ${ }^{2}$, and A. Raichoor ${ }^{1,3}$ \\ ${ }^{1}$ Laboratoire AIM (UMR 7158 CEA/DSM-CNRS-Université Paris Diderot), Irfu/Service d'Astrophysique, CEA-Saclay, \\ 91191 Gif-sur-Yvette Cedex, France \\ e-mail: chaty@cea.fr \\ 2 UJF-Grenoble 1/CNRS-INSU, Institut de Planétologie et d'Astrophysique de Grenoble (IPAG) UMR 5274, 38041 Grenoble, France \\ 3 GEPI, Observatoire de Paris-Meudon, 5 place Jules Janssen, 92195 Meudon, France
}

Received 15 August 2010 / Accepted 15 February 2011

\section{ABSTRACT}

\begin{abstract}
Context. Microquasars are accreting Galactic sources that are also observed to launch relativistic jets. A key signature of the ejection is non-thermal radio emission. The level of this jet component at high frequencies is still poorly constrained.

Aims. The X-ray binary and microquasar black hole candidate XTE J1550-564 exhibited a faint X-ray outburst in April 2003 during which it stayed in the X-ray low/hard state. We took optical and near-infrared (NIR) observations with the ESO/NTT telescope during this outburst to distinguish the various contributions to the spectral energy distribution (SED) and investigate the presence of a jet component.

Methods. Photometric and spectroscopic observations allowed us to construct an SED and also to produce a high time-resolution lightcurve.

Results. The SED shows an abrupt change of slope from the NIR domain to the optical. The NIR emission is attributed to non-thermal synchrotron emission from the compact, self-absorbed jet that is known to be present in the low/hard state. This is corroborated by the fast variability, colours, lack of prominent spectral features and evidence for intrinsic polarisation. The SED suggests the jet break from the optically thick to the thin regime occurs in the NIR.

Conclusions. The simultaneous optical-NIR data allow an independent confirmation of jet emission in the NIR. The transition to optically thin synchrotron occurs at NIR frequencies or below, which leads to an estimated characteristic size $\gtrsim 2 \times 10^{8} \mathrm{~cm}$ and magnetic field $\lesssim 5 \mathrm{~T}$ for the jet base, assuming a homogeneous one-zone synchrotron model.
\end{abstract}

Key words. accretion, accretion disks - X-rays: individuals: XTE J1550-564 - binaries: close - ISM: jets and outflows infrared: stars - X-rays: binaries

\section{Introduction}

X-ray binary systems are composed of a companion star and a compact object - a black hole or a neutron star. In low-mass $\mathrm{X}$-ray binaries (LMXBs), the companion star is a late-type star filling its Roche lobe. Matter transiting through the Lagrange point forms an accretion disk around the compact object. The LMXBs spend most of their time in a quiescent state with a low X-ray luminosity. Outbursts occasionally occur, owing to an instability in the accretion disk, during which the X-ray luminosity increases by several orders of magnitude. Those LMXBs that additionally show non-thermal radio emission that is sometimes spatially resolved into jets are called microquasars (see e.g. Chaty 2006; Chaty \& Bessolaz 2006; Fender 2006; Mirabel \& Rodríguez 1998).

Several canonical states for LMXBs have been defined according to their X-ray emission properties (see e.g. Belloni 2010; Remillard \& McClintock 2006), the main ones being:

- the high/soft state, characterized in the X-rays by a high luminosity, dominated by the thermal emission of the accretion disk, with a peak temperature $\sim 1-1.5 \mathrm{keV}$, emitting as a multicolour blackbody from optical to X-rays;

- the low/hard state, with the X-rays dominated by a powerlaw component, the accretion disk being weak in the X-ray

* Based on observations collected at the European Southern Observatory, Chile, through programs 071.D-0071 and 079.D-0623. band, at a temperature $\sim 0.01-0.5 \mathrm{keV}$; the hard state is invariably associated with strong, flat spectrum radio emission that is attributed to a compact, self-absorbed jet (with an extension of $\sim 10^{-6 \prime \prime}$, see e.g. Fender 2006);

- the quiescent state, when the X-ray luminosity is low and the optical/infrared emission is dominated by the emission of the companion star.

We also point out the existence of the intermediate state. During the transition between the low/hard and the high/soft state, discrete ejections are most of the time observed in radio, with an extension $\sim 0.1-10^{\prime \prime}$ (see e.g. Fender 2006).

XTE J1550-564 was discovered as a transient X-ray binary in September 1998 by the All Sky Monitor (ASM) onboard the Rossi-XTE satellite (Remillard et al. 1998). Optical (Orosz et al. 1998; Jain et al. 1999) and radio (Campbell-Wilson et al. 1998) counterparts were promptly identified, classifying XTE J1550-564 as a microquasar. The companion star is in a 1.541 day orbit (Jain et al. 2001b). It has been shown spectroscopically to be a G8 IV-K4 III at a distance of $5.3 \mathrm{kpc}$; the compact object, with a mass of $10.5 \pm 1.0 M_{\odot}$, is a black hole candidate (Orosz et al. 2002). Five main outbursts have been observed in XTE J1550-564 (see Fig. 1 left part). The first one, which started in September 1998 and lasted about 200 days, was the most powerful: at its maximum, the $2-10 \mathrm{keV}$ flux reached almost $500 \mathrm{ASM}$ counts/s (=6.8 Crabs). During this outburst, the source had a complex behaviour and transited through all 

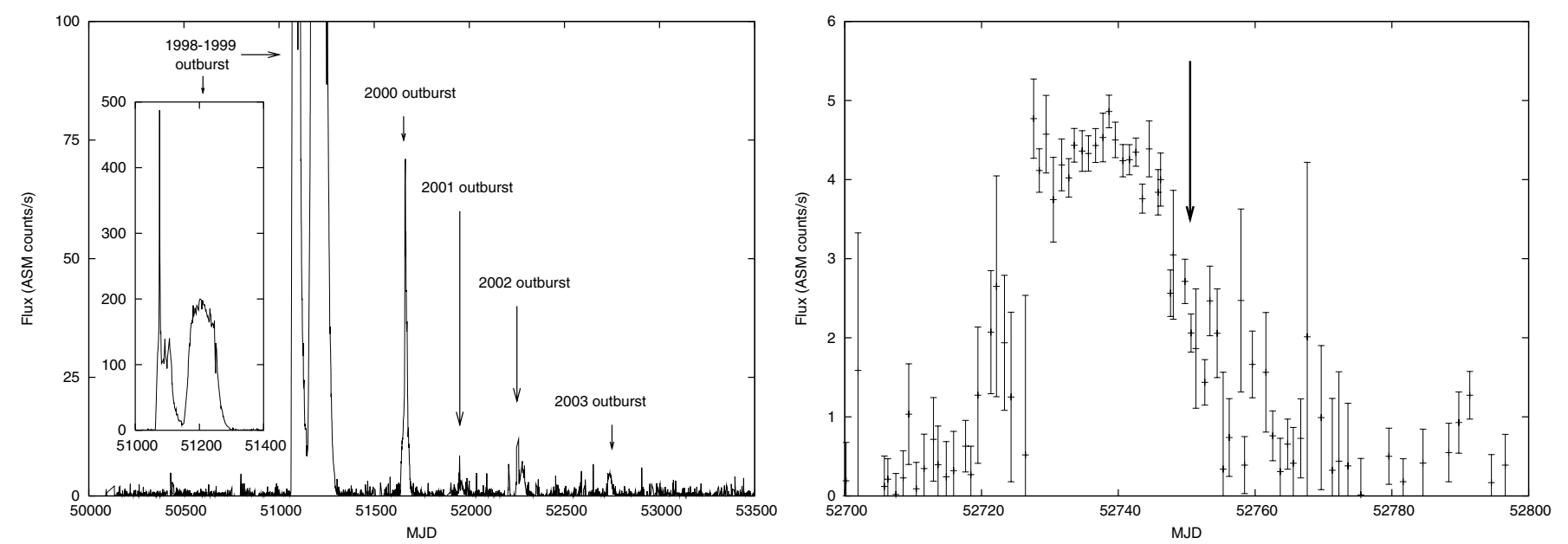

Fig. 1. Lightcurves of the flux of the microquasar XTE J1550-564 in the 2-10 keV band acquired by ASM/Rossi-XTE. Left: lightcurve from 1996 to 2005. Right: zoom during the 2003 outburst. The date of our observations (2003 April 21) is marked by the arrow.

canonical X-ray spectral states (Homan et al. 2001). In 2000, the source exhibited another outburst, the $2-10 \mathrm{keV}$ flux reaching around $1 \mathrm{Crab}$, and the source transiting again through different spectral states. In 2001, 2002, and 2003, the source showed three less powerful outbursts, during which it remained in the low/hard state (Aref'ev et al. 2004; Sturner \& Shrader 2005). Such "mini" outbursts following a major eruption have been seen in other X-ray binaries (e.g. Šimon 2010) and in some dwarf novae (Kuulkers et al. 1996). The mechanism triggering these mini-outbursts is not understood within the framework of the standard LMXB disk instability model (Dubus et al. 2001). They could be related to the accretion disk becoming eccentric during outburst in binary systems with small mass ratios (Hellier 2001).

Overlapping contributions from the companion star, the accretion disk, and from the relativistic jet make the optical to near-infrared (NIR) wavelength range particularly important to study. For instance, a compilation of optical and NIR observations of the black hole candidate X-ray binary GX 339-4 showed that its NIR emission during low/hard states was non-thermal, likely synchrotron radiation emanating from the compact jet of this microquasar (Corbel \& Fender 2002). Its spectral energy distribution (SED) showed the typical signature of a compact jet, namely a clear change in slope in the optical-NIR domain with an inverted power-law at lower frequencies. Similar signatures have been seen in other LMXBs (Kalemci et al. 2005; Migliari et al. 2006).

Here, we report on the results of optical and NIR observations of the microquasar XTE J1550-564 during the 2003 outburst. XTE J1550-564 remained in the low-hard state during the outburst. Our goal was to assess whether emission from the compact jet extended to the NIR. The weakness of the X-ray ouburst made it potentially more favourable to detect a jet contribution, because the accretion luminosity is expected to decrease faster than the jet luminosity (Heinz \& Sunyaev 2003). The optical and NIR observations and data reduction are described in Sect. 2. The results are presented in Sect. 3 and discussed in Sect. 4.

\section{Observations and data reduction}

\subsection{Photometry and spectroscopy}

Our observations were performed during the night between 2003 April 21st and 22nd (Fig. 1 right shows when our observations took place during the X-ray outburst), using the NTT (New
Technology Telescope) telescope on the La Silla observatory of ESO (European Southern Observatory), as part of a Target of Opportunity (ToO) programme (PI S. Chaty). They took place on the declining phase of the 2003 outburst of XTE J1550-564 and consist of optical and NIR photometry (both deep and rapid), NIR spectroscopy and polarimetry. The results from the NIR polarimetry were reported in Dubus \& Chaty (2006).

The NIR data were obtained in the $J, H$, and $K_{\mathrm{s}}$ filters with the spectro-imager SoFI (Son oF Isaac), using the large field imaging (field of view of 4.92 $\times 4$ t.92 $^{\prime}$ and image scale of 0.'288/pixel). For each filter of the NIR deep photometric observations, we observed the source at nine different positions with $60 \mathrm{~s}$ exposure time each, to estimate and substract the thermal sky emission, with a standard shift-and-combine jitter procedure. We also performed rapid photometry in the $K_{\mathrm{s}}$ filter, observing the source at different positions for three hours, with an integration time of $2 \mathrm{~s}$ for each exposure. The readout mode was double correlated read, leading to an overhead time of $\sim 50 \%$; therefore we reached a time resolution of nearly $3 \mathrm{~s}$ for this rapid photometry.

To calibrate the photometric observations, we observed two photometric standard stars of the Persson catalogue (Persson et al. 1998): sj 9136 and sj9146. Concerning the NIR spectroscopy, we took 24 spectra of $60 \mathrm{~s}$ each, half with the blue grism $(0.95-1.64 \mu \mathrm{m})$ and half with the red grism $(1.53-2.52 \mu \mathrm{m})$; we also took spectra of the telluric standard Hip 63689 to correct the spectra of XTE J1550-564 from the atmospheric absorption.

The optical data were obtained in the $B, V, R, I$ and $Z$ filters with the spectro-imager EMMI (Extraordinaire Multi-Mode Imager), using the large field imaging (field of view of 9'. $\times$ 9 '. 1 , binning $2 \times 2$ for a better sensitivity and image scale of 0 .'332/pixel). We observed the source for $300 \mathrm{~s}$ in the $B$-band, for $3 \mathrm{~s}$ and $60 \mathrm{~s}$ in the $V$-band and for $60 \mathrm{~s}$ in the $R-, I-$ and $Z$-band. We also observed the photometric standard stars PG 1633 and PG 1657. We performed rapid photometry in the $V$ filter (a series of $10 \mathrm{~s}$ exposures during two hours). Concerning the optical spectroscopy, we took three spectra of $300 \mathrm{~s}$ each and a spectrum of a spectro-photometric standard star (LTT 7379).

We used the Image Reduction and Analysis Facility (IRAF) suite to perform data reduction, carrying out standard procedures of optical and NIR image reduction, including flat-fielding and NIR sky subtraction. For the standard photometry, we used a 
Table 1. Characteristic extinction coefficients at La Silla and derived zero-points for the different optical and NIR bands.

\begin{tabular}{lcc}
\hline \hline Filter & ext. & $Z_{\mathrm{p}}$ \\
\hline$B$ & 0.214 & $0.069 \pm 0.002$ \\
$V$ & 0.125 & $-0.584 \pm 0.002$ \\
$R$ & 0.091 & $-0.741 \pm 0.003$ \\
$I$ & 0.051 & $-0.231 \pm 0.003$ \\
$J$ & 0.08 & $2.062 \pm 0.010$ \\
$H$ & 0.03 & $2.232 \pm 0.006$ \\
$K_{\mathrm{s}}$ & 0.05 & $2.799 \pm 0.008$ \\
\hline
\end{tabular}

median filter before carrying out aperture photometry with the noao.daophot package. We obtained the apparent magnitudes $m_{\text {app }}$ from the instrumental magnitudes $m_{\text {inst }}$ through the following formula, where $Z_{\mathrm{p}}$ is the zero-point, ext the extinction coefficient, and airmass the airmass at the time of the observations: $m_{\text {app }}=m_{\text {inst }}-Z_{\mathrm{p}}-$ ext $\times$ airmass.

We used the characteristic extinction coefficients at La Silla and obtained the zero points given in Table 1 by averaging the values obtained with the different standard stars. For the $Z$ filter, the apparent magnitudes of the standard stars were not available, so we did not calibrate $m_{\text {app }}(Z)$.

Concerning the spectra, we used the IRAF noao.twodspec package to extract the spectra and perform wavelength calibration. Since we did not observe any spectro-photometric standard stars, we did not perform flux calibration. We divided the NIR spectra of XTE J1550-564 by the spectra of the telluric standard and multiplied it by the spectra of a $4600 \mathrm{~K}$ blackbody (we took the average of the effective temperature range of XTE J1550-564 companion star given by Orosz et al. 2002: between $4100 \mathrm{~K}$ and $5100 \mathrm{~K}$ ). We point out that the signal-tonoise ratio of the optical spectra of XTE J1550-564 was too faint to securely identify any feature. Fortunately the NIR spectra were more exploitable, but not in the whole waveband coverage though, due to absorption.

We give in Fig. 2 a finding chart in NIR wavelengths for XTE J1550-564.

\subsection{Polarimetry}

We also performed polarimetric observations on August 1 and 2, 2007. We collected a series of $10 \mathrm{~s} K_{\mathrm{s}}$ band exposures of the field around XTE J1550-564 at the ESO NTT using SOFI in polarimetric mode. A Wollaston prism splits the incoming light into two images with perpendicular polarisation. XTE J1550-564 was jittered along the mask for sky subtraction. Images taken at four different angles were used to compensate for the instrumental polarisation and this was checked against observations of unpolarised standards (see Dubus \& Chaty 2008, for details).

In addition, we also used polarimetric observations to derive the $K_{\mathrm{s}}$ magnitude in quiescence. Since we did not observe any photometric standard star during this run, we performed relative photometric calibration, thanks to isolated and bright stars of the 2MASS catalogue (Cutri et al. 2003), close to XTE J1550-564. We obtained the following apparent magnitude for XTE J1550-564: $\mathrm{K}_{s}=16.25 \pm 0.05 \mathrm{mag}$.

\section{Results}

\subsection{Photometry and extinction}

We have estimated the interstellar absorption using the column density on the line of sight derived from Chandra observations: $N_{\mathrm{H}}=0.88 \pm 0.1 \times 10^{22} \mathrm{~cm}^{-2}$ (Corbel et al. 2006). This value

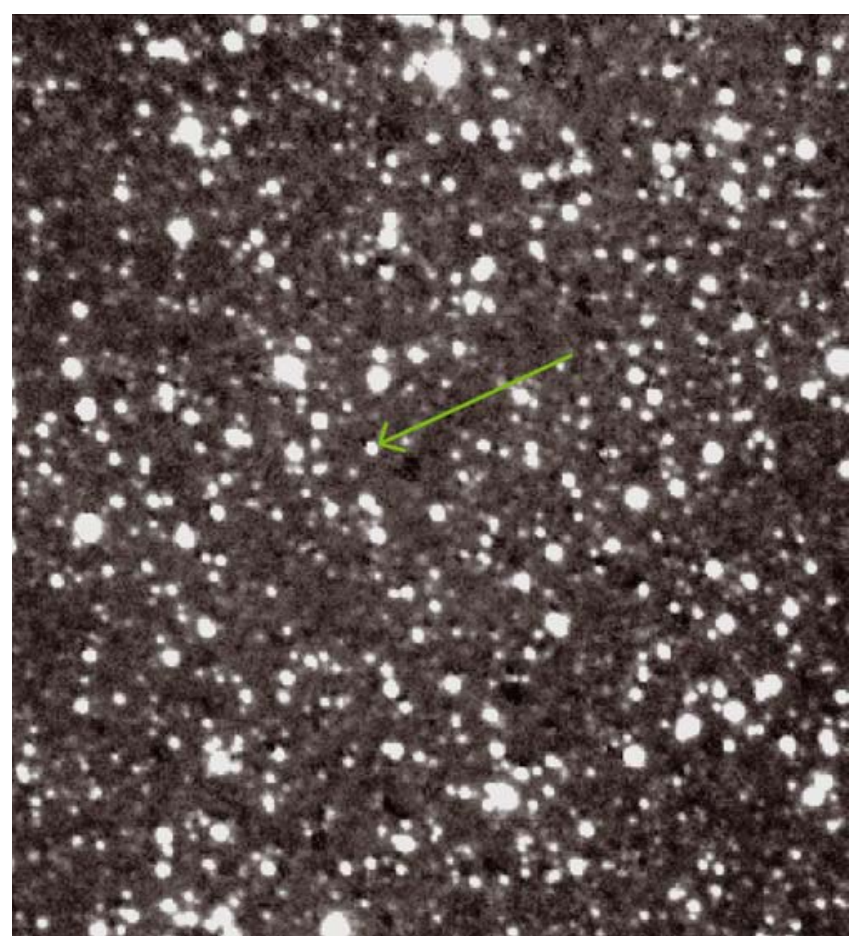

Fig. 2. XTE J1550-564 field of view $\left(3.9^{\prime} \times 3.9^{\prime}, H\right.$ filter $)$ : north is to the top and East to the left. The XTE J1550-564 counterpart is indicated by the arrow.

Table 2. Apparent magnitudes, interstellar absorption, and dereddened apparent magnitudes for various wavelengths.

\begin{tabular}{lccc}
\hline \hline Filter & $m_{\mathrm{app}}$ & $A_{\lambda}$ & $m_{\mathrm{app}}-A_{\lambda}$ \\
\hline$B$ & $20.00 \pm 0.04$ & $6.57 \pm 0.75$ & $13.43 \pm 0.79$ \\
$V$ & $18.48 \pm 0.03$ & $4.92 \pm 0.56$ & $13.56 \pm 0.59$ \\
$R$ & $17.35 \pm 0.01$ & $3.69 \pm 0.42$ & $13.56 \pm 0.43$ \\
$I$ & $16.33 \pm 0.01$ & $2.36 \pm 0.27$ & $13.97 \pm 0.28$ \\
$J$ & $14.50 \pm 0.01$ & $1.39 \pm 0.16$ & $13.11 \pm 0.17$ \\
$H$ & $13.46 \pm 0.02$ & $0.94 \pm 0.11$ & $12.52 \pm 0.13$ \\
$K_{\mathrm{s}}$ & $12.40 \pm 0.01$ & $0.56 \pm 0.06$ & $11.84 \pm 0.07$ \\
\hline
\end{tabular}

is somewhat lower than, but still consistent with, the HI column density integrated through the whole Galaxy, given by both the Leiden/Argentine/Bonn $\left(N_{\mathrm{H}}=1.01 \times 10^{22} \mathrm{~cm}^{-2}\right)$ and Dickey \& Lockman $\left(N_{\mathrm{H}}=0.897 \times 10^{22} \mathrm{~cm}^{-2}\right)$ surveys. The interstellar absorption in the $V$-band $A_{V}$ is then deduced from the relation $A_{v}=5.59 \times 10^{-22} N_{\mathrm{H}}$ (Predehl \& Schmitt 1995) and the different $A_{\lambda}$ using the relations established by Cardelli et al. (1989). Table 2 lists the apparent magnitudes we derived from our observations, together with the values obtained for $A_{\lambda}$ (taking into account the $1.6 \sigma$ uncertainty on $N_{\mathrm{H}}$ ) and, finally, the dereddened apparent magnitudes (taking into account the uncertainty on $A_{\lambda}$ ). The observed fluxes before and after corrections are plotted in the left part of Fig. 3 .

There is clearly a change of slope in between the NIR and visible wavelengths. The reddened NIR and optical spectral slopes are consistent with powerlaws of spectral index 1.7 and 3.9 respectively, and the dereddened NIR and optical with spectral index of 0.3 and -1.3 respectively ${ }^{1}$. The best-fitting powerlaws are indicated in Fig. 3 (left) by dotted lines.

In order for both the NIR and optical slopes to be roughly compatible, one would have to decrease the value of the column

${ }^{1}$ The spectral index $\alpha$ is defined as $F_{v} \propto v^{-\alpha}$. 

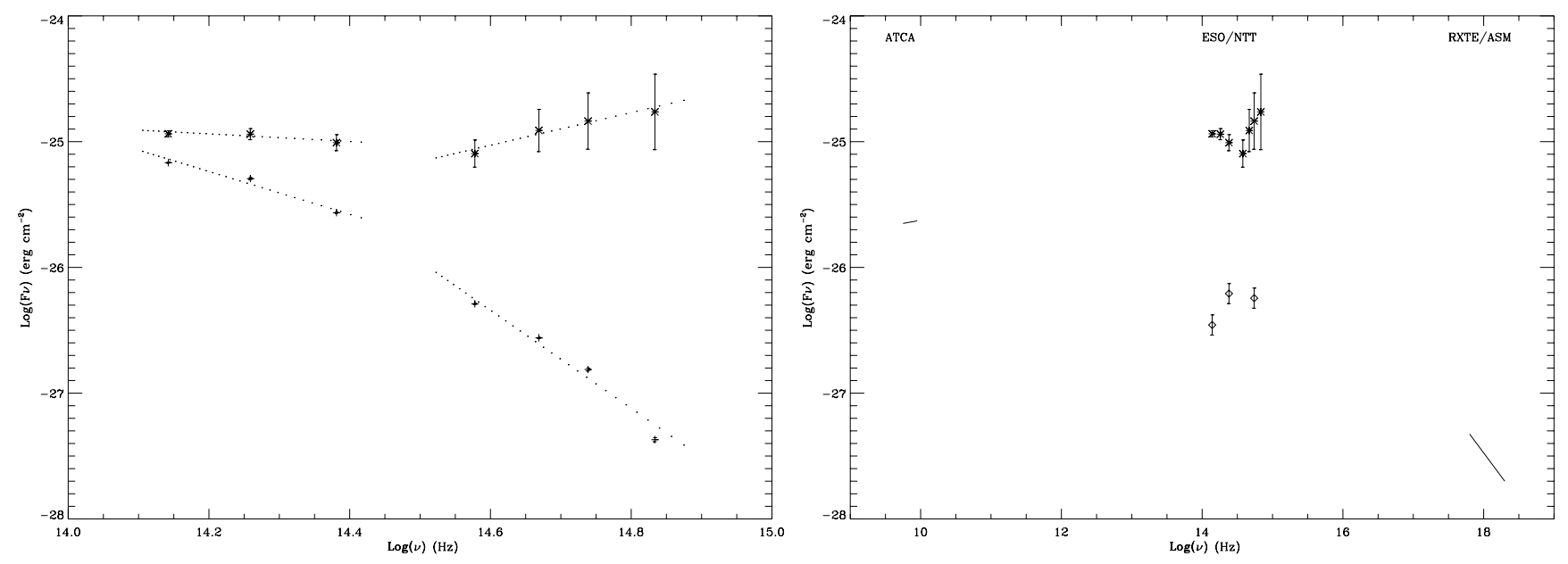

Fig. 3. XTE J1550-564 SEDs during the 2003 mini-outburst. Left: comparison between reddened (crosses in the lower part) and dereddened (asterisks in the upper part) optical/NIR SEDs. From optical (right) to IR (left): BVRI JHK points. The error bars are also shown and are bigger in the case of the dereddened points owing to the $1.6 \sigma$ uncertainty on the $N_{\mathrm{H}}$ and then on $A_{v}$ (see Sect. 3.1). We also show here power law slopes, with spectral indices given in the text. Right: broadband SED. Our ESO/NTT observations taken during the mini-outburst are shown with asterisk symbols, and the data taken during quiescence ( $K_{\mathrm{s}}$ flux obtained during our polarimetric run, $J$ and $V$ flux from Orosz et al. 2011) are shown with lozenges. All these data are dereddened with $A_{V}=4.92 \mathrm{mag}$, and the error bars show the uncertainty on the column density. We also plot the simultaneous X-ray flux/spectrum (Aref'ev et al. 2004), and the ATCA radio fluxes observed during the 2002 outburst, with similar X-ray flux.

density to $A_{V}=3.5$ (corresponding to $N_{\mathrm{H}} \sim 0.6 \times 10^{22} \mathrm{~cm}^{-2}$ ), which is clearly well below the value derived from X-ray observations, and also from the HI surveys, including uncertainty. And even by doing this, the optical I magnitude point is never aligned with the NIR and optical slopes, confirming that a change of slope is clearly present between the optical and NIR power laws, with two different spectral indices. Because the NIR spectrum is optically thin with a positive spectral index, the jet break must be located either in the NIR (around the H-band as suggested by right panel of Fig. 3), or towards longer wavelengths, in the MIR domain. Only contemporaneous observations from optical to MIR domain would allow us to constrain the exact location of the jet break.

\subsection{SED}

The broad-band SED of XTE J1550-564 from radio to X-rays is shown in the right part of Fig. 3, including our optical/NIR observations taken during the mini-outburst and also during quiescence. The optical/NIR data are dereddened from interstellar absorption. The line in the lower right indicates the flux and spectral index of the simultaneous X-ray data of XTE J1550-564 during the 2003 mini-outburst, as observed by ASM/Rossi-XTE (Aref'ev et al. 2004). We also include radio data obtained during the 2002 mini-outburst with a similar X-ray flux, reported with the line in the left. Although no contemporary radio observations could be found, this is indicative of the radio emission that could have been expected from the compact jet during the low/hard X-ray mini-outburst of 2003 (see Sect. 1).

The optical data are consistent with the Rayleigh-Jeans tail of a multicolour blackbody, which is characteristic of the emission coming from the outer part of the accretion disk, whereas the NIR data suggest a non thermal, inverted spectra, which is characteristic of synchrotron emission and is usually associated with a compact radio jet (see e.g. Corbel \& Fender 2002, for GX 339-4 and Chaty et al. 2003, for XTE J1118+480). The level of NIR emission is consistent with the extrapolation to high frequencies of the (non-contemporary) flat/inverted radio spectrum and the extrapolation to low frequencies of the X-ray spectrum (with photon index $\approx 1.6$, Aref'ev et al. 2004; Sturner \& Shrader 2005).

\subsection{Colour-magnitude diagrams}

The absolute magnitudes of XTE J1550-564 during the minioutburst are reported in the colour-magnitude diagrams (CMD) in Fig. 4. The absolute magnitude was computed via

$M_{\lambda}=m_{\lambda}+5-5 \times \log (d(\mathrm{pc}))-A_{\lambda}$.

In both CMDs, the big asterisk indicates the position of XTE J1550-564 optical/NIR counterpart, and the small asterisks surrounding it represent the parameter space of the source, taking into account the uncertainty on its distance $(d=5.3 \pm$ $2.3 \mathrm{kpc}$, Orosz et al. 2002) and on the column density $\left(N_{\mathrm{H}}=\right.$ $0.88 \pm 0.1 \times 10^{22} \mathrm{~cm}^{-2}$, Corbel et al. 2006, as reported in Table 2). According to Orosz et al. (2002), the companion star has a type from G8 IV to K4 III, hence the quiescent state lies in the lower left part of the red giant branch. We indicate in Fig. 4 (right) the position of the XTE J1550-564 quiescent magnitudes.

The NIR CMD (Fig. 4 right part) shows that the source is redder in outburst than in quiescence. The companion star contributes more flux to the $J$ band than to the $K_{\mathrm{s}}$ band. Hence, the significantly redder colour implies an additional contribution with a flat or inverted spectrum.

The optical CMD (Fig. 4 left part) shows that the source was bluer in these bands in outburst compared to quiescence, which is consistent with the dominant contribution of the accretion disk in the optical. Indeed, when a multicolour blackbody spectrum is evolving with a higher flux and temperature, the flux increases more in the $B$-band than in the $V$-band, which implies a decrease in the $(B-V)$ colour.

Both CMDs confirm that the optical and NIR fluxes evolve differently between the mini-outburst and quiescence. 

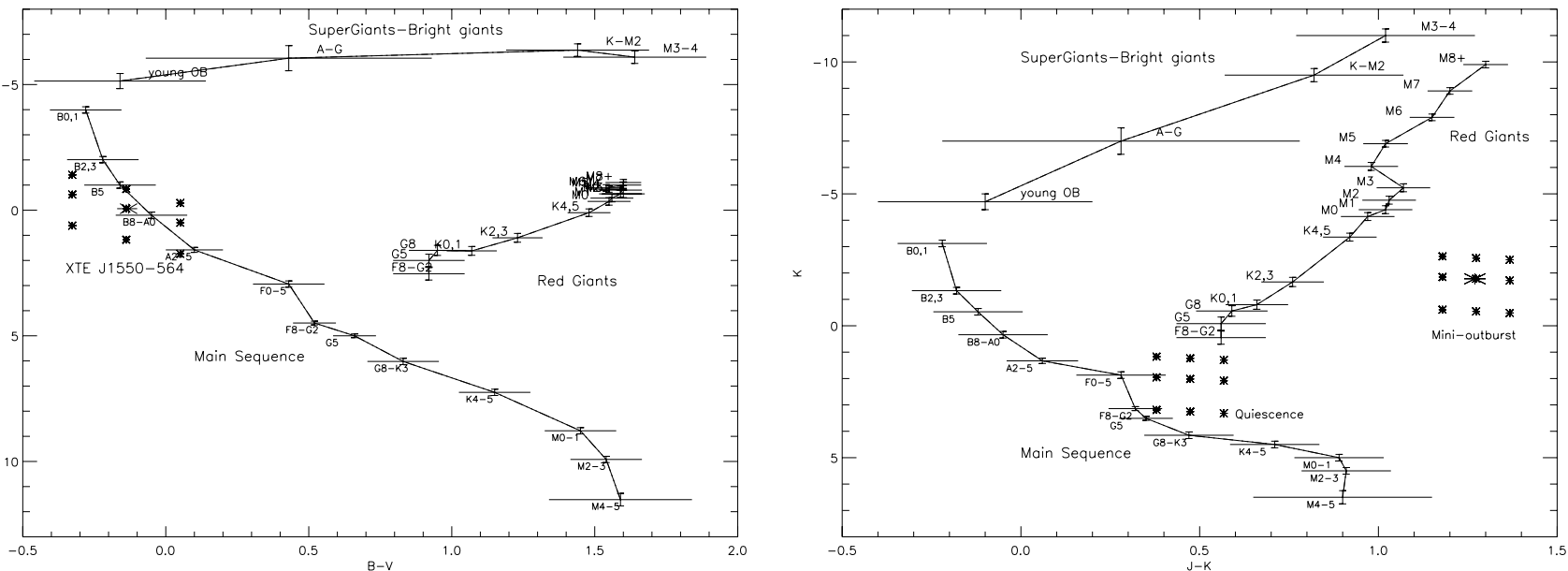

Fig. 4. CMDs with characteristic absolute magnitudes of various spectral types, on which we overplot our optical and NIR data (left and right plot respectively) for XTE J1550-564 during the 2003 mini-outburst; the nine asterisks represent the position of XTE J1550-564 counterpart, taking into account the uncertainties on its distance and column density. The bigger central asterisk indicates its position using the best fitted distance and column density. In the NIR CMD we also add XTE J1550-564 position corresponding to quiescence, using magnitudes from this paper and Orosz et al. (2011).
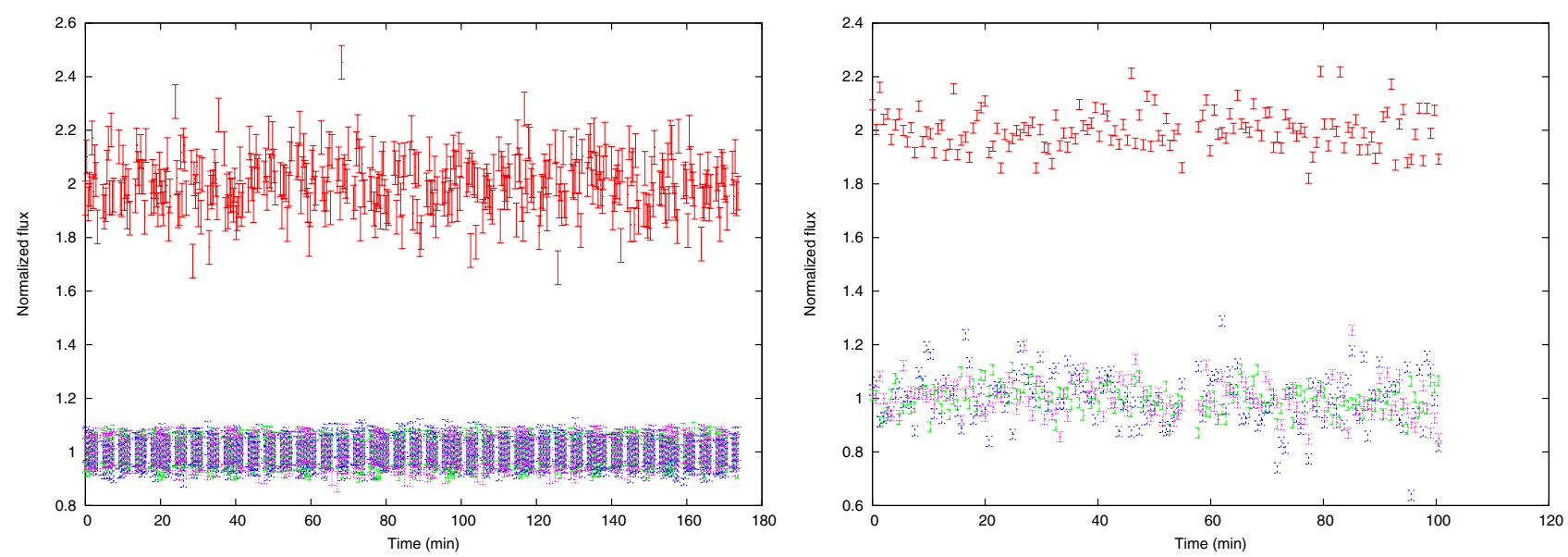

Fig. 5. Rapid photometry lighcurve in the $K_{\mathrm{s}}$ filter (left) and $V$ filter (right): corrected and normalized flux of XTE J1550-564 (red, artificially increased by 1 unit) and of stars of the field of view with similar brightness (green, blue, purple respectively).

\subsection{Rapid photometry}

We performed rapid photometry in the $V$ and $K_{\mathrm{s}}$ filters on XTE J1550-564 and on stars present in the field of view that were of comparable brightness to XTE J1550-564. We then averaged the fluxes of these stars to get a mean flux, and we finally divided the flux of XTE J1550-564 by this mean flux. These corrected and normalized fluxes are shown in Fig. 5 in the optical and NIR respectively, where we can see the intrinsic variations of the X-ray source and the surrounding stars. While rapid photometry in the NIR is rarely performed on LMXBs, it allows us to constrain rapid phenomena occuring on short timescale, either in the accretion disk, or related to the jet.

The rapid photometry shows that the source presents variations of amplitude in the NIR greater than those of the surrounding stars, whereas in the optical, the source behaviour is comparable with the surrounding stars. The standard deviations of this optical and NIR rapid photometry for XTE J1550-564 and the surrounding stars are presented in Table 3. Again, this suggests that the origin of the NIR and optical emission are different. However, note that variations in the optical of a similar
Table 3. Standard deviations taken from the rapid photometry lightcurves of XTE J1550-564 and of various stars from the field of view.

\begin{tabular}{ll|ll}
\hline \hline \multicolumn{2}{c}{$K_{\mathrm{s}}$ filter } & \multicolumn{2}{c}{$V$ filter } \\
\hline XTE J1550-564 & 0.099 & XTE J1550-564 & 0.074 \\
Star \#1 & 0.020 & Star \#1 & 0.051 \\
Star \#2 & 0.024 & Star \#2 & 0.065 \\
Star \#3 & 0.021 & Star \#3 & 0.098 \\
\hline
\end{tabular}

Notes. The stars have a brightness similar to the one of XTE J1550-564.

amplitude to those in the NIR would probably not have been detected with these observations.

\subsection{Spectroscopy}

We took two IR spectra in the blue and red grisms, both are very absorbed, with a low $\mathrm{S} / \mathrm{N}$ ratio. As shown in Fig. 6, we only detect a faint emission line corresponding to $\mathrm{Br} \gamma$ transition at $2.166 \mu \mathrm{m}$, very likely produced by the accretion disk. Apart 


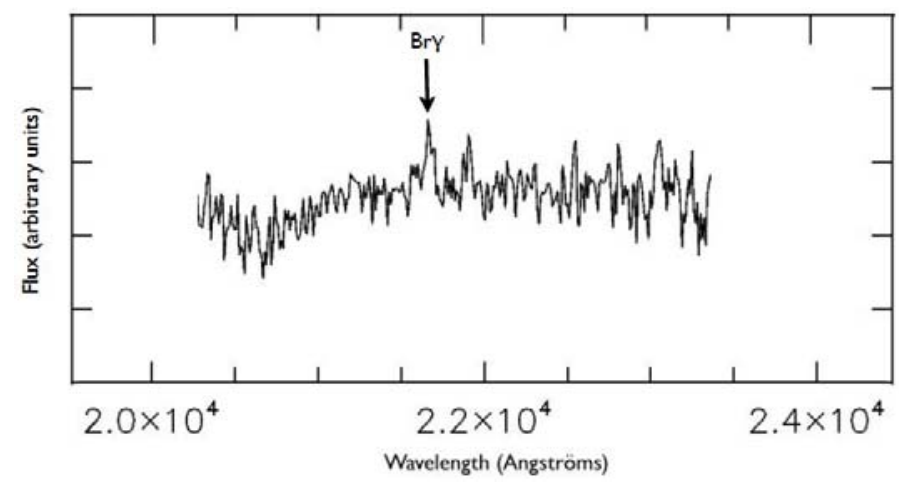

Fig. 6. Corrected NIR spectra of XTE J1550-564 (the $y$-axis is in arbitrary units). Apart from the faint $\operatorname{Br} \gamma$ emission line visible at $2.166 \mu \mathrm{m}$, both blue and red grisms are featureless.

from this, both NIR spectra are featureless, consistent with nonthermal emission emanating from the compact jet.

\subsection{Polarimetry}

The NIR polarimetry of XTE J1550-564, taken on the same night as part of the same observing programme, was reported earlier (Dubus \& Chaty 2006). The polarimetry showed an excess polarisation in XTE J1550-564 compared with other stars in the field-of-view. Here, we report on polarimetric observations collected well after the mini-outburst described in this paper. The goal was to obtain a better measure of the polarisation signal from field stars in order to find the absolute value of the NIR polarisation from XTE J1550-564 during its 2003 mini-outburst.

As described in Sect. 2.2, XTE J1550-564 was detected in quiescence at a magnitude $K_{\mathrm{s}}=16.25 \pm 0.05 \mathrm{mag}$, but the low $\mathrm{S} / \mathrm{N}$ prevented a meaningful polarisation measurement. The mean polarisation of the bright stars within 1' of XTE J1550-564 (see right panel of Fig. 1 in Dubus \& Chaty 2006) was found to $\mathrm{be} \approx 1.4 \%$. The corresponding reduced Stokes parameter values are $q=-0.8 \pm 0.3 \%$ and $u=1.1 \pm 0.5 \%$ with the errors derived from the scatter of the stars $q$ and $u$ values. Assuming the polarisation of the field stars has not changed between 2003 and 2007, we correct for this mean polarisation and find that the absolute $K_{\mathrm{s}}$ band polarisation during the 2003 mini-outburst of XTE J1550-564 was about 2.4\%. This confirms that the polarisation fraction was in excess of the interstellar polarisation that could be expected with $E(B-V)=0.7(\approx 0.7 \%$, see Dubus $\&$ Chaty 2006). We were unable to determine the angle correction. The few polarised standards available to this effect are very bright. Several were observed by defocusing the telescope, but the resulting photometry proved too unreliable to be of use. Hence, we cannot give the orientation of the polarisation angle with respect to the jet axis.

\section{Discussion}

\subsection{The spectral break}

We detected a break between the NIR and optical wavelengths during a mini-outburst of XTE J1550-564, with a positive spectral index in NIR, suggesting optically thin emission consistent with a jet spectrum. The break is clearly visible in the spectral energy distribution and colour-magnitude diagrams. Inaccuracies in the column density used to deabsorb the fluxes cannot account for this break. The NIR spectrum is featureless, apart from a faint $\operatorname{Br} \gamma$ emission line. The $K_{\mathrm{s}}$ lightcurve shows $\approx 10 \%$ variability on short timescales, which is suggestive of a non-thermal component. Evidence for an intrinsic IR polarisation during the outburst also points towards synchrotron emission (Dubus \& Chaty 2006). The overall SED is reminiscent of the low/hard state SED of GX 339-4 and XTE J1118-480 where the infrared emission was attributed to the compact jet (see Sect. 1). Our data lead us to conclude that synchrotron jet emission dominated in the NIR during the 2003 mini-outburst of XTE J1550-564.

A dominant jet contribution in NIR was also put forward by Russell et al. (2010) to explain the correlations between the $H$ band and X-ray fluxes during the 2000 outburst of XTE J1550-564. The 3-10 keV flux during our observations was about $6 \times 10^{-10} \mathrm{erg} \mathrm{cm}^{-1} \mathrm{~s}^{-1}$. Comparing our results with Fig. 1 of Russell et al. (2010), we find that with $H=13.46$ the source was in NIR about $50 \%$ brighter during our observations than during the decline of the 2000 outburst, at the time when the source reached the same X-ray flux. Our observations are consistent with the picture of an increasing jet contribution in the NIR as the source becomes harder and fainter in X-rays (Russell et al. 2006).

The $V$ flux in the 2003 mini-outburst is brighter than that seen in 2000 for similar X-ray luminosities (Jain et al. 2001a; Russell et al. 2010). The optical spectrum we measure is compatible with a Rayleigh-Jeans tail. This requires the temperature of the outer disk radius to be $\gtrsim 10^{4} \mathrm{~K}$. Otherwise, the flat part of the disk blackbody spectrum $\left(F_{v} \propto v^{1 / 3}\right)$ should be visible ${ }^{2}$. Here, we assume the temperature distribution as a function of disk radius $R$ is $T_{\text {disk }} \propto R^{-3 / 4}$, which is adequate for an accretion disk in outburst (Dubus et al. 2001). The temperature is high enough to ionize hydrogen in the outer disk, as expected in outburst. The data during the 2000 decline did not show a break in the spectra between $H, I$ and $V$ bands. The weaker $V$ flux in 2000 may have been due to a hotter disk temperature: this would have placed the Rayleigh-Jeans tail at higher frequencies.

\subsection{Physical conditions at the jet base}

The infrared fluxes decrease progressively from $12.0 \mathrm{mJy}$ in $K$ to $6.6 \mathrm{mJy}$ in $I$ before increasing again. The spectral steepening suggests the transition in the compact jet to optically thin synchrotron emission occurs at IR frequencies. The optically thin emission is dominated by the emission from the innermost region in self-absorbed jet models (Blandford \& Konigl 1979; Hjellming \& Johnston 1988; Kaiser 2006). This is usually the case because the optically thin flux along the jet decreases rapidly with distance. The turnover frequency decreases along the jet and the summed contribution produces the flat spectrum at lower frequencies.

Assuming the innermost region with cross-section radius $R_{0}$ and length $H_{0}$ is seen sideways, the transition from thick to thin synchrotron emission occurs at $\tau_{v}=\alpha_{v} R_{0} \approx 1$. The synchrotron emission and absorption coefficients have analytical expressions for a power-law distribution of electrons with an index $p$. Further assuming that the energy density in non thermal electrons is a fraction $\xi$ of the magnetic energy density $B_{0}^{2} / 8 \pi$ in the region, this gives a relationship between the peak frequency, $\xi$, $R_{0}$ and $B_{0}$. The approximate flux at the peak frequency can be

\footnotetext{
2 Steeper accretion disk spectra can be expected when the disk is irradiated (Hynes 2005) but this is unlikely to be the case here given the weak X-ray flux in outburst.
} 
derived and depends on $\xi, R_{0}, H_{0}$ and $B_{0}$. We find the following relationships for $B_{0}$ and $R_{0}$ (see Appendix A)

$$
\begin{aligned}
& B_{0} \approx 5 \times 10^{4} v_{14} S_{10}^{-1 / 9} \xi^{-2 / 9} h^{1 / 9} d_{5}^{-2 / 9} \mathrm{G} \\
& R_{0} \approx 2.5 \times 10^{8} v_{14}^{-1} S_{10}^{17 / 36} \xi^{-1 / 18} h^{-17 / 36} d_{5}^{17 / 18} \mathrm{~cm}
\end{aligned}
$$

where we have taken a minimum Lorentz factor for the electrons $\gamma_{\min }=1, p=2.5$ (optically thin spectral index of 0.75 ), $v_{\text {peak }}=10^{14} v_{14} \mathrm{~Hz}, S_{\text {peak }}=10 S_{10} \mathrm{mJy}, d=5 d_{5} \mathrm{kpc}$ and $H_{0}=h R_{0}$. Similar results are obtained for $p=2$ or $p=3$. These equations apply equally to black hole or neutron star LMXBs. The magnetic field depends most sensitively on the turnover frequency.

Self-absorbed models usually make the assumption that adiabatic cooling is dominant over the radiative timescales. The adiabatic timescale is $t_{\text {ad }} \gtrsim R_{0} / c \approx 8 \mathrm{~ms}$. The synchrotron timescale is $t_{\text {sync }} \propto \gamma^{-1} B_{0}^{-2} \approx 120 \gamma^{-1} \mathrm{~ms}$ with the magnetic field derived above. Since NIR emission requires electron Lorentz factors $\gamma \approx 20$, it means that this is marginally verified at the jet base. We find variability on a few second timescales. $K$ band variability on $\approx 200 \mathrm{~ms}$ timescales was also reported in GX 339-4 during a low/hard state (Casella et al. 2010) but the above suggests that there could be NIR variability down to $10 \mathrm{~ms}$ timescales. Beyond the jet base, $t_{\text {sync }} / t_{\text {ad }}$ increases rapidly because $B \sim z^{-1}$ along the jet axis $z$ whereas $R \sim z^{\beta}$ with $\beta \approx 0.5$ to reproduce flat spectra (e.g. Hjellming \& Johnston 1988; Kaiser 2006). Self-Compton cooling can be ignored because a luminosity ratio of Compton to synchrotron emission $L_{\mathrm{ic}} / L_{\mathrm{sync}} \approx 0.2$ is inferred using the $R_{0}$ and $B_{0}$ given above. It can be shown that $L_{\text {ic }} / L_{\text {sync }} \propto v_{\text {peak }} S_{\text {peak }}^{-5 / 18}$. Synchrotron self-Compton emission from the jet base will be negligible unless $v_{\text {peak }}$ moves into the visible.

Russell et al. (2010) speculate that the X-ray emission in the hard state becomes fully jet-dominated when the $3-10 \mathrm{keV}$ flux is below a few $10^{-10} \mathrm{erg} \mathrm{cm}^{-2} \mathrm{~s}^{-1}$. Our observations do show that the NIR emission lies close to the extrapolated X-ray spectrum. If the cutoff at $\approx 100 \mathrm{keV}$ in INTEGRAL is caused by synchrotron emission (but see Zdziarski \& Gierliński 2004), then the maximum electron Lorentz factor is $\gamma_{\max } \approx 6000$. The X-ray emitting electrons should be radiatively cooled and the X-ray spectrum below the cutoff should have a photon index $\gtrsim 2$ when an index $\approx 1.7$ is observed.

Finally, a magnetic field of a few teslas at the jet base is inevitable regardless of the detailed model if the NIR break is due to self-absorption. For comparison, the equipartition magnetic field with thermal pressure in the accretion disk at a radius close to the compact object is

$B_{\text {eq }} \approx 5 \times 10^{7} \eta^{-1 / 2} M_{1}^{-1 / 2} \dot{m}^{1 / 2} r^{-5 / 4} \mathrm{G}$,

where $r$ is the radius in units of the last stable orbit, $M_{1}$ is the mass of the compact object in solar masses, $\dot{m}$ is the accretion rate in units of Eddington and $\eta=H / R \rightarrow 1$ for Bondi-Hoyle or radiatively inefficient accretion. The X-ray luminosity during our observations is $\approx 10^{-3} L_{\text {Edd }}$, which implies $\dot{m} \gtrsim 10^{-3}$. Therefore, the magnetic field at the jet base in XTE J1550-564 represents at most $1 \%$ of the equipartition magnetic field at the innermost radius.

Curiously, compact jets in AGNs typically have turnovers in the 1-100 GHz range (Kellermann \& Pauliny-Toth 1981), exactly as expected if the ratio $B / B_{\text {eq }}$ were constant from microquasars to quasars. If this ratio is also constant in an object, then the turnover frequency will move to longer wavelengths as the mass accretion rate decreases. This can be tested observationally in microquasars when they decline from outbursts.

\section{Conclusion}

We have obtained simultaneous NIR to optical coverage of the microquasar XTE J1550-564 during a mini-outburst. Our dataset shows a break in the SED from the NIR to the optical. The optical emission is compatible with the Rayleigh-Jeans tail of the accretion disk. The lack of prominent spectral feature in the NIR, the fast variability and the evidence for intrinsic polarisation lead us to attribute the NIR emission to synchrotron radiation from the compact jet. Based on correlations between IR and X-ray fluxes during its 2000 outburst, Russell et al. (2010) also interpreted the NIR emission from XTE J1550-564 as jet emission. Evidence for NIR or optical jet emission from XTE J1550-564 was also suggested by Jain et al. (2001a); Corbel et al. (2001); Russell et al. (2007). The NIR luminosity represents about $1.7 \%$ of the X-ray luminosity. The jet contribution appears to be more important, in terms of the NIR to X-ray ratio, during the faint 2003 mini-outburst than during the 2000 outburst.

The SED shows a steepening from $K$ to $I$, suggesting the transition from optically thick to thin synchrotron emission occurs around $10^{14} \mathrm{~Hz}$. If this interpretation is correct, then the magnetic field at the jet base is at most a few teslas, or about $1 \%$ of the equipartition magnetic field in the accretion disk close to the black hole as in AGN compact jets. The NIR emission region must be small and sub-second variability can be expected.

Our data provide only a snapshot of the SED during an outburst. The evolution of the jet break during an outburst can provide important diagnostics of the jet physics (Heinz \& Sunyaev 2003; Markoff et al. 2003). Good sampling of the optical to NIR SED both in time and frequency, ideally in combination with polarisation measurements, is required to identify this break independently of the radio or X-ray observations and to test models that suggest jet emission can dominate the X-ray emission.

Acknowledgements. S.C. thanks the ESO staff for performing service observations, and S.C. and G.D. are grateful to an anonymous referee who helped to improve the paper. IRAF is distributed by the National Optical Astronomy Observatories, which are operated by the Association of Universities for Research in Astronomy, Inc., under a cooperative agreement with the National Science Foundation. Rossi-XTE Results were provided by the ASM/Rossi-XTE teams at MIT and at the Rossi-XTE SOF and GOF at NASA's GSFC. This research has made use of NASA's Astrophysics Data System Bibliographic Services. This publication makes use of data products from the Two Micron All Sky Survey, which is a joint project of the University of Massachusetts and the Infrared Processing and Analysis Center/California Institute of Technology, funded by the National Aeronautics and Space Administration and the National Science Foundation. This work was supported by the Centre National d'Études Spatiales (CNES), based on observations obtained with MINE - the Multiwavelength INTEGRAL NEtwork -; and by the European Community via contract ERC-StG-200911.

\section{Appendix A: Magnetic field and size of the jet base}

We assume that the synchrotron-emitting region at the jet base is a homogeneous cylinder of radius $R_{0}$ and height $H_{0}=h R_{0}$. The electrons follow a power-law distribution $\mathrm{d} N=K_{0} \gamma^{-p} \mathrm{~d} \gamma$; the magnetic field is $B_{0}$. The standard formula for the synchrotron absorption coefficient $\alpha_{v}$ is (Rybicki \& Lightman 1979)

$$
\alpha_{v}=\frac{\sqrt{3} e^{3}}{8 \pi m_{\mathrm{e}}^{2} c^{2}}\left(\frac{3 e}{2 \pi m_{\mathrm{e}} c}\right)^{\frac{p}{2}} \Gamma\left(\frac{3 p+2}{12}\right) \Gamma\left(\frac{3 p+22}{12}\right) K_{0} B_{0}^{\frac{p+2}{2}} v^{-\frac{p+4}{2}} .
$$


Similarly, the optically thin emissivity $j_{v}$ is

$j_{v}=\frac{\sqrt{3} e^{3}}{2 \pi m_{\mathrm{e}} c^{2}}\left(\frac{m_{\mathrm{e}} c}{3 e}\right)^{-\frac{p-1}{2}} \Gamma\left(\frac{3 p+19}{12}\right) \Gamma\left(\frac{3 p-1}{12}\right) \frac{K_{0} B_{0}^{\frac{p+1}{2}}}{p+1} v^{-\frac{p-1}{2}}$

so that the flux from the region can be written as $S_{v}=$ $(1 / 2)\left(R_{0} / d\right)^{2} H_{0} j_{v}$, with $d$ the distance to the source. The synchrotron self-absorbed emission peaks at the frequency $v_{\text {peak }}$ where $\tau_{v}=\alpha_{v} R_{0} \approx 1$. The jet emission transits from the flat optically thick part to the optically thin part at $v_{\text {peak }}$, regardless of the detailed emission further down the jet (which only affects emission at frequencies below $v_{\text {peak }}$ ). Inverting $S_{v}=S_{\text {peak }}$ and $\tau_{\text {peak }}=1$ gives two equations on $R_{0}$ and $B_{0}$, as functions of $S_{\text {peak }}, v_{\text {peak }}, h, p$ and $K_{0}$. We assume the energy in non-thermal electrons $\epsilon_{e}$ is a fraction $\xi$ of the magnetic field energy density

$\epsilon_{e} \equiv \int_{\gamma_{\min }}^{\gamma_{\max }} K_{0} \gamma^{1-p} m_{\mathrm{e}} c^{2} \mathrm{~d} \gamma=\xi \frac{B_{0}^{2}}{8 \pi}$.

$K_{0}$ can be expressed as a function of the equipartition fraction $\xi, B_{0}, p, \gamma_{\min }$ and $\gamma_{\max }$. We have assumed $p=2.5$ and $\gamma_{\max } \gg$ $\gamma_{\min }=1$ in deriving Eq. (2).

\section{References}

Aref'ev, V. A., Revnivtsev, M. G., Lutovinov, A. A., \& Sunyaev, R. A. 2004, Astron. Lett., 30, 669

Belloni 2010, The Jet Paradigm, Lecture Notes in Physics (Berlin: Springer Verlag), 794

Blandford, R. D., \& Konigl, A. 1979, ApJ, 232, 34

Campbell-Wilson, D., McIntyre, V., Hunstead, R., et al. 1998, IAU Circ., 7010, 3

Cardelli, J. A., Clayton, G. C., \& Mathis, J. S. 1989, ApJ, 345, 245

Casella, P., Maccarone, T. J., O’Brien, K., et al. 2010, MNRAS, 404, L21

Chaty, S. 2006, in Proc. Frontier Objects in Astrophysics and Particle Physics, Vulcano Workshop, May 22-27, ed. G. Giovannelli, \& F. Mannocchi (Italian Physical Society), 93, 329

Chaty, S., \& Bessolaz, N. 2006, A\&A, 455, 639

Chaty, S., Haswell, C. A., Malzac, J., et al. 2003, MNRAS, 346, 689

Corbel, S., \& Fender, R. P. 2002, ApJ, 573, L35
Corbel, S., Kaaret, P., Jain, R. K., et al. 2001, ApJ, 554, 43

Corbel, S., Tomsick, J. A., \& Kaaret, P. 2006, ApJ, 636, 971

Cutri, R. M., Skrutskie, M. F., van Dyk, S., et al. 2003, 2MASS All Sky Catalog of point sources, NASA/IPAC Infrared Science Archive

Dubus, G., \& Chaty, S. 2006, A\&A, 458, 591

Dubus, G., \& Chaty, S. 2008, in Blazar Variability across the Electromagnetic Spectrum, ed. PoS, Vol. BLAZARS2008, 37

Dubus, G., Hameury, J., \& Lasota, J. 2001, A\&A, 373, 251

Fender, R. 2006, Jets from X-ray binaries, ed. W. H. G. Lewin, \& M. van der Klis (Cambridge University Press), 381

Heinz, S., \& Sunyaev, R. A. 2003, MNRAS, 343, L59

Hellier, C. 2001, PASP, 113, 469

Hjellming, R. M., \& Johnston, K. J. 1988, ApJ, 328, 600

Homan, J., Wijnands, R., van der Klis, M., et al. 2001, ApJS, 132, 377

Hynes, R. I. 2005, ApJ, 623, 1026

Jain, R. K., Bailyn, C. D., Orosz, J. A., McClintock, J. E., \& Remillard, R. A. 2001a, ApJ, 554, L181

Jain, R. K., Bailyn, C. D., Orosz, J. A., et al. 2001b, ApJ, 546, 1086

Jain, R. K., Bailyn, C. D., Orosz, J. A., Remillard, R. A., \& McClintock, J. E. 1999, ApJ, 517, L131

Kaiser, C. R. 2006, MNRAS, 367, 1083

Kalemci, E., Tomsick, J. A., Buxton, M. M., et al. 2005, ApJ, 622, 508

Kellermann, K. I., \& Pauliny-Toth, I. I. K. 1981, ARA\&A, 19, 373

Kuulkers, E., Howell, S. B., \& van Paradijs, J. 1996, ApJ, 462, L87

Markoff, S., Nowak, M., Corbel, S., Fender, R., \& Falcke, H. 2003, A\&A, 397, 645

Migliari, S., Tomsick, J. A., Maccarone, T. J., et al. 2006, ApJ, 643, L41

Mirabel, I. F., \& Rodríguez, L. F. 1998, Nature, 392, 673

Orosz, J., Bailyn, C., \& Jain, R. 1998, IAU Circ., 7009, 1

Orosz, J. A., Groot, P. J., van der Klis, M., et al. 2002, ApJ, 568, 845

Orosz, J. A., Steiner, J. F., McClintock, J. E., et al. 2011, ApJ, 730, 75

Persson, S. E., Murphy, D. C., Krzeminski, W., Roth, M., \& Rieke, M. J. 1998, AJ, 116, 2475

Predehl, P., \& Schmitt, J. 1995, A\&A, 293, 889

Remillard, R. A., \& McClintock, J. E. 2006, ARA\&A, 44, 49

Remillard, R., Morgan, E., McClintock, J., \& Sobczak, G. 1998, IAU Circ., 7019, 1

Russell, D. M., Fender, R. P., Hynes, R. I., et al. 2006, MNRAS, 371, 1334

Russell, D. M., Maccarone, T. J., Körding, E. G., \& Homan, J. 2007, MNRAS, 379,1401

Russell, D. M., Maitra, D., Dunn, R. J. H., \& Markoff, S. 2010, MNRAS, 405, 1759

Rybicki, G. B., \& Lightman, A. P. 1979, Radiative Processes in Astrophysics, ed. G. B.Rybicki, \& A. P. Lightman (Wiley-Interscience)

Sturner, S. J., \& Shrader, C. R. 2005, ApJ, 625, 923

Šimon, V. 2010, A\&A, 513, A71

Zdziarski, A. A., \& Gierliński, M. 2004, Progress of Theoretical Physics Supplement, 155, 99 